DOI: 10.1002/adfm.((please insert DOI)

\title{
Anomalous oxidation states in multilayers for fuel cell applications
}

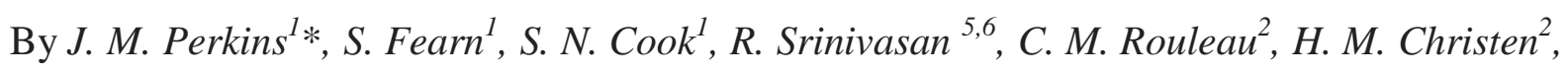
G. D. West ${ }^{3}$, R. J. H. Morris ${ }^{4}$, H. L. Fraser ${ }^{5}$, S.J. Skinner ${ }^{1}$, J A Kilner ${ }^{1}$ and D W McComb ${ }^{1 *}$. Corresponding Author $(s)^{*}$

[*] Dr J. M. Perkins, Dr S. Fearn, S. N. Cook, Dr S. J. Skinner, Prof. J. A. Kilner, Prof. D. W. $\mathrm{McComb}$

Department of Materials, Imperial College London. SW7 2AZ (UK)

j.m.perkins@imperial.ac.uk

d.mccomb@imperial.ac.uk

Dr. R. Srinivasan, Prof. H. L. Fraser

Department of Materials Science and Engineering,

Ohio State University, Columbus, Ohio. (USA)

Dr C. M. Rouleau, Dr H. M. Christen,

Center for Nanophase Materials Sciences,

Oak Ridge National Laboratory, Oak Ridge, TN. 37831 (USA)

Dr G. D. West

Loughborough University,

Loughborough, Leicestershire. (UK)

Dr R. J. H. Morris 
University of Warwick,

Coventry, Warwickshire, CV4 7AL. (UK)

Keywords: STEM, EELS, SIMS, fuel cell, PLD,

Significant recent interest has been directed towards the relationship between interfaces and reports of enhanced ionic conductivity. To gain a greater understanding of the effects of hetero-interfaces on ionic conductivity advanced analytical techniques including electron microscopy (TEM/STEM), electron energy loss spectroscopy (EELS), and secondary ion mass spectrometry (SIMS) have been used to characterize $\mathrm{CeO}_{2} / \mathrm{Ce}_{0.85} \mathrm{Sm}_{0.15} \mathrm{O}_{2}$ multilayer thin films grown by pulsed laser deposition. High quality growth is observed, but ionic conductivity measured by impedance spectroscopy and ${ }^{18} \mathrm{O}$ tracer experiments is consistent with bulk materials. EELS analysis reveals the unusual situation of layers containing only $\mathrm{Ce}(\mathrm{IV})$ adjacent to layers containing both $\mathrm{Ce}(\mathrm{III})$ and $\mathrm{Ce}(\mathrm{IV})$. Post oxygen annealing induced oxygen diffusion and mixed oxidation states in both layers, but only in the vicinity of low angle grain boundaries perpendicular to the layers. The implications of this remarkable metastability of Ce oxidation states on the design of novel electrolytes for solid oxide fuel cells is discussed.

\section{Introduction}

Intermediate Temperature Solid Oxide Fuel Cells (ITSOFC's) are poised to make an important contribution towards the twin goals of reducing carbon emissions, and security of supply through the implementation of high efficiency distributed electricity generation. An important thread in the 
development of (ITSOFCs) has been the continued improvement of both mixed and ionic conductors that show high levels of oxygen ion transport at relatively low temperatures $\left(\leq 500^{\circ} \mathrm{C}\right) .{ }^{[1]}$ The initial goal was optimisation of bulk and grain boundary ionic conductivity to give good low temperature performance from conventional thick film and ceramic materials. An example of this work is the optimisation of the bulk (lattice) oxygen ion conductivity in the fluorite structured oxides. These materials have been used as solid electrolytes ever since the discovery of oxide ion conductivity in ceramics over 100 years ago. ${ }^{[2]}$ One of the advantages of these materials comes from the ease of creation of oxygen vacancies by aliovalent doping, and their high mobility in the fluorite structure. ${ }^{[3]}$ The key to obtaining good low temperature bulk conductivity is to minimize the activation energy for conductivity, and this may be done by carefully size matching aliovalent substitution cations which lead to mobile extrinsic oxygen vacancies. ${ }^{[4]}$ Minimising the activation energy has not been easy for zirconia based materials, hence there has been a drive towards development of analogous materials, such as the $\mathrm{CeO}_{2}$ based electrolytes ${ }^{[5]}$ and other low temperature materials such as lanthanum gallate. ${ }^{[6]}$ These electrolytes have higher ionic conductivities than the commonly used yttria stabilised zirconia (YSZ) electrolyte ${ }^{[2,7,8]}$ but at significantly lower temperatures.

The optimisation of the grain boundary conductivity has been more difficult because of the complex interplay of impurities, segregation, and space charge effects, which more often than not lead to a degradation of the conductivity in conventional materials. The specific influence of microstructure on ionic transport has therefore become of great interest. There have been many reports on the effects of increasing the interfacial density by moving to nanocrystalline materials and it is apparent that both the electronic and ionic conductivity can be enhanced by the presence of these homointerfaces. ${ }^{[9,10]}$ However, isotope exchange studies carried out by DeSouza et al ${ }^{[11]}$ showed that no enhanced oxygen diffusion was observed in nano-YSZ. Indeed, it was shown that oxygen transport and diffusion kinetics were impaired by the increased number of grain boundaries in this material. These results imply therefore that ionic conductivity would not be enhanced in nano-YSZ. 
In other nano-sized multilayer structures such as $\mathrm{CaF}_{2}-\mathrm{BaF}_{2}$ enhanced interfacial ion transport was observed for structures with layer thicknesses in the range 10-80nm, whereas for smaller layer thicknesses conductivity was decreased. ${ }^{[12]}$ The enhanced conductivity was explained by space charge considerations, whereas the reduced conductivity was observed to be due to a columnar structure formed during growth.

Korte $^{[13]}$ and co-workers have investigated the effects of elastic strain at heterojunctions in highly substituted oxide systems, where space charge effects can be negligible, and have concluded that conductivity can be both enhanced and depressed by the effects of elastic strain at the interfaces. Experimental observations of increased ionic conductivities have been reported for highly oriented epitaxial structures of several electrolyte materials such as YSZ, ${ }^{[2,13]} \mathrm{Sm}_{2} \mathrm{O}_{3}$-doped $\mathrm{CeO}_{2},{ }^{[5,9]}$ and

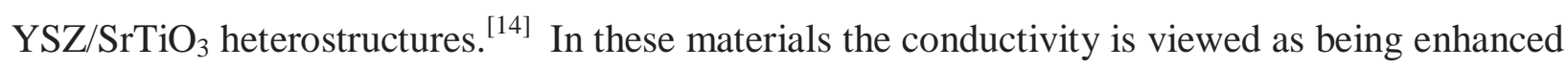
through engineering of the interfaces, rather than manipulation of the bulk chemical composition. In this contribution, an extensive study of the structure-property relationships in $(\mathrm{Sm}, \mathrm{Ce}) \mathrm{O}_{2-\mathrm{x}} / \mathrm{CeO}_{2}$ multilayers grown on an $\mathrm{MgO}$ substrate are reported as a potential system that might exhibit anomalously high ionic conductivity. ${ }^{[15-17]}$ The use of doped ceria in ion conducting applications is well known, ${ }^{[7]}$ however the incorporation of selectively doped layers leads to well defined interface formation between doped and undoped regions. The specific $(\mathrm{Sm})$ system was originally part of a larger selection of cation dopant systems to be investigated due to preliminary reports by Kosacki et al. of the potential for enhancement by thin film multilayer compositions. ${ }^{[16]}$ Here we use a combination of techniques to investigate the extent of ionic transport in a heterostructured $\mathrm{CeO}_{2} / \mathrm{Ce}_{0.85} \mathrm{Sm}_{0.15} \mathrm{O}_{2-\mathrm{x}}$ thin film and comment on the complex and unexpected properties of this multilayer system.

\section{Results}




\subsection{Conductivity}

Figure 1(a) shows a typical impedance spectrum obtained from the multilayer systems. In general, the samples exhibit what appears to be a single semicircle which upon closer inspection is seen to deviate somewhat from being symmetrical at low frequencies. These are similar to those reported previously in fluorite structured oxide thin films. ${ }^{[2,17]}$ The majority of the semicircle can be fitted using an equivalent circuit consisting of a single resistor and constant phase element (CPE) in a parallel arrangement. The low frequency region can be more accurately fitted when utilizing an additional resistor and CPE in series. The equivalent circuit used to fit this data is inset in Fig. 1(a). Multilayer samples with the repeat unit of $20 \mathrm{~nm} \mathrm{CeO}_{2} / 30 \mathrm{~nm} \mathrm{Ce}_{0.85} \mathrm{Sm}_{0.15} \mathrm{O}_{2-\mathrm{x}}$ and 50, 100, 200 and 400 cycles thereof were investigated. The capacitance of the first constant phase element (CPE1), calculatedfrom the CPE-T value, was $\sim 10^{-13} \mathrm{~F}$ for all tested samples. ${ }^{[18]}$ This is of the same order of magnitude as the capacitance that can be estimated from the dimensions of the samples and the relative permittivities of the materials of which they comprise $\left(\varepsilon_{\mathrm{r}}=10^{1}-10^{2}\right)$, and R1 is attributed to the behavior of the doped layers due to the low conductance of the magnesia substrate and the undoped ceria layers. CPE2 was typically on the order of $10^{-10} \mathrm{~F}$ and could correspond to the behavior of the low angle grain boundaries discussed later. The conductivity results can be seen in Fig. 2, and exhibit an Arrhenius relationship with activation energies of $\sim 0.72 \mathrm{eV} . \mathrm{MgO}$ substrates were used as they have negligible ionic conductivity; this was confirmed by acquiring impedance data from a pure $\mathrm{CeO} 2$ thin film grown on $\mathrm{MgO}$ under identical conditions to the multilayer samples. The samples show highly consistent temperature dependent conductivity behaviour especially in samples with cycles numbering greater than 100. The conductivity is shown to be somewhat lower than that of bulk samarium doped ceria of the same stoichiometry with identical activation energies.

\subsection{Microstructural Characterisation}


Bright field (BF) and high angle annular dark field (HAADF) images of the as-grown sample are shown in Fig. 3. The intensity in the HAADF image is dominated by the average atomic number $(\mathrm{Z})$ i.e. - Rutherford scattering - and is approximately proportional to $Z^{2}{ }^{[20]}$ Thus, bright layers correspond to the samaria doped ceria (SDC) layers, and the dark layers to the undoped $\mathrm{CeO}_{2}$ layers. Comparison with the scale bar shows that the doped and undoped layers are close to the targeted thicknesses of $30 \mathrm{~nm}$ and $20 \mathrm{~nm}$, respectively.

Comparison of the BF and HAADF images, which were acquired simultaneously, shows that contrast is opposite in the two images as expected. However, features in the BF image that might be interpreted as crystalline grains within the layers can also be observed. In addition, features that cross the interface between doped and undoped layers, not parallel to the growth direction, are also present. These features can be identified in the HAADF image (indicated by circles). Such features in HAADF images are often ascribed to surface damage/amorphisation or implantation from the TEM sample preparation method. This was not expected as the preparation route concluded with a low energy $(<200 \mathrm{~V})$ argon ion milling designed to remove surface damage. The lack of an amorphised layer at the edge of the sample confirms the effectiveness of this procedure. Furthermore, the high resolution HAADF image confirms high quality epitaxial growth across the multilayers, (Fig. 3c).

A TEM image from a similar region of the sample is shown in Fig. 4. The growth direction is shown on the image and the interfaces are marked for clarity. The features observed in the STEM images that appear to cross the multilayer interfaces can also be identified in Fig. 4, and appear to be more prevalent in the doped than the undoped layers. A high resolution TEM image of one of these features is shown in Fig. 5. It is clear that the feature corresponds to a low angle grain boundary aligned along the growth direction. The arrows denote the direction of the lattice fringes and illustrate the misorientation between the two grains. The boundary plane is not well oriented with respect to the incident electron beam so is not easily identified - the boundary region is indicated by 
dotted lines. Extensive investigations revealed that the majority of the features observed in the STEM and TEM images correspond to low angle grain boundaries, the majority of which are approximately aligned with the growth direction. The small misorientation explains why these GBs had little influence on the observed RHEED and minimal influence on the XRD results (other than broadening the 002 rocking curves to $\sim 1$ degree). These may be associated with the origin of the second component of the AC impedance spectrum. Such defects have been reported to have a detrimental effect on ionic conductivity of a material. ${ }^{[2]}$ Energy-dispersive x-ray (EDX) spectroscopy mapping supports the evidence for good quality, compositionally abrupt layers seen in the HAADF images (Fig. 6). The HAADF image in Fig. 6(b) shows a region analysed by EDX mapping that contains a low angle grain boundary. The Sm and Ce compositional maps obtained from an EDX spectrum image of the region, Fig. 6(c) and (d) show that there is no evidence for compositional changes associated with the low angle grain boundary.

Electron energy-loss spectroscopy (EELS) was used to probe the oxygen K-, cerium $\mathrm{M}_{4,5^{-}}$and samarium $\mathrm{M}_{4,5}$-edges in the multilayers (detailed in supplemental information). Compositional profiles extracted from the region indicated on the image are shown in Fig. 7. The probe size was < $0.5 \mathrm{~nm}$ and the distance between each point in the line profile was $\sim 1 \mathrm{~nm}$. Based on these profiles the average interface width between the doped and undoped layers is $\sim 2 \mathrm{~nm}$ which is consistent with the HAADF images and EDX maps. Since the sample thickness is essentially constant in the region analysed the relative change in each of the profiles is a reliable indication of the compositional variation. In the case of the Sm profile the signal decreases to essentially zero in the undoped region confirming that no inter-diffusion has taken place. The Ce signal in the doped region is $20 \%$ less than in the undoped region. This corresponds to the expected level of doping. The oxygen signal in the doped region is about $17 \%$ less than in the doped region. A large number of areas on as-grown multilayer samples have been analysed and a decrease in the oxygen signal is typically found to be in the range $15-20 \%$. On the assumption that one oxygen vacancy is created for every two Ce(IV) 
cations that are replaced by Sm(III) cations, then 15 at\% Sm-doping should result in a decrease of $3.75 \%$ in the oxygen signal relative to the undoped layer i.e. $\mathrm{Ce}_{0.85} \mathrm{Sm}_{0.15} \mathrm{O}_{1.925} \mathrm{c} / \mathrm{w} \mathrm{CeO}_{2}$. Therefore, compositional variations cannot be used to explain the high oxygen vacancy concentration. This calculation assumes that the Ce oxidation state in the doped layer is $\mathrm{Ce}(\mathrm{IV})$ which is inconsistent with the decrease in oxygen content measured. In order to accommodate a $15 \%$ decrease in oxygen, the composition of the doped layer would have to be $\left(\mathrm{Ce}^{\mathrm{IV}}\right)_{0.2}\left(\mathrm{Ce}^{\mathrm{III}}\right)_{0.65}\left(\mathrm{Sm}^{\mathrm{III}}\right)_{0.15} \mathrm{O}_{1.6}$. In order to check for compositional homogeneity within each layer, total Ce compositional profiles were extracted from spectrum images recorded in the doped and undoped layers (Fig. 8). The variation within each layer is approximately $+/-5 \%$.

Multiple linear least squares (MLLS) fitting of the $\mathrm{Ce}_{4,5}$-edges was used to extract the $\mathrm{Ce}(\mathrm{III}) / \mathrm{Ce}(\mathrm{IV})$ ratio. ${ }^{[21]}$ Spectra from $\mathrm{CeO}_{2}$ and $\mathrm{CePO}_{4}$ powders were used as reference spectra for Ce(IV) and Ce(III), respectively (Fig. 9). ${ }^{[22]}$ The MLLS routine is more robust if all of the spectra have the same energy resolution. Where this is not the case, the data should be interpolated or smoothed. The absolute energy scale may not be correct due to drift of the elastic peak from the calibrated scale. To overcome this, each spectrum image was calibrated using the spectrum that was most similar to one of the standards. An example of the result of the fit is shown in Fig. 10. The valence profiles, $\mathrm{Ce}^{\mathrm{IV}} /\left(\mathrm{Ce}^{\mathrm{III}}+\mathrm{Ce}^{\mathrm{IV}}\right)$, extracted from the spectrum images can be correlated with the HAADF signal facilitating differentiation between compositional and structural contrast in the images.

For the as-grown sample in Fig. 10, the valence ratio varies systematically across the multilayer and closely correlates with the multilayer structure. It is clear that the undoped layers which are darker in the HAADF image contain Ce(IV) only. In contrast, the Sm doped layers contain both $\mathrm{Ce}(\mathrm{III})$ and $\mathrm{Ce}(\mathrm{IV})$ in the ratio of approximately 30:70. There are contrast features in the image that show some correlation with changes in the valence profile. 
The results of MLLS fitting of profiles acquired within each of the doped and undoped layers are shown in Fig. 8. These results confirm that there is essentially only one oxidation state in the undoped layer and that the doped layer contains Ce in both oxidation states. However, it is also clear that the absolute ratio differs from that obtained previously, and moreover, there is significant variation within the doped layer. There does not appear to be a strong correlation between the HAADF signals and the valence profiles.

In order to correlate the STEM-EELS results with the conductivity measurements, experiments were carried out on a sample that had been annealed at $800^{\circ} \mathrm{C}$ in air. The results from MLLS fitting of spectrum images from this sample are shown in Fig. 11. The profile acquired across the multilayer structure (A) is quite different to that obtained from the as-grown sample (Figs. 8 and 10) in that both the doped and the undoped layers now contain a mixture of $\mathrm{Ce}(\mathrm{III})$ and $\mathrm{Ce}(\mathrm{IV})$, although the $\mathrm{Ce}(\mathrm{IV})$ content remains higher in the undoped layer. This is further confirmed by the profile within the undoped layer (B) which shows a Ce(IV) level in the range $55 \%$ to $82 \%$. This large variation shows some correlation with intensity variations in the HAADF profile. This weak correlation is also observed in the doped region (C).

In the case of ceria it might have been expected that after heating in air at $800^{\circ} \mathrm{C}$ (for at least two hours) all Ce(III) ions would have been fully oxidized to Ce(IV). This is not the case which implies that there is a barrier to diffusion. Instead it appears that some oxygen has diffused from the doped layer into the undoped layer, resulting in the reduction of $\mathrm{Ce}$ in the undoped layer and oxidation of some $\mathrm{Ce}$ in the doped layer (which was also observed in the ${ }^{18} \mathrm{O}$ diffusion experiment). It is believed that the origin of the contrast within the layers is associated with low angle grain boundaries. Although these boundaries are approximately aligned with the growth direction, the boundary plane may be at any angle to the incident electron beam direction and the contrast in the image may only arise from certain regions that are oriented to enhance channeling in the Z-contrast image. It is hypothesized that these low angle grain boundaries act as preferential diffusion channels for oxygen 
ions to move between the layers and initiate a redox process. Where the boundary is oriented appropriately some correlation between the valence profile and HAADF signal will be observed. The Ce(IV)-rich regions spaced inhomogeneously along both the doped and undoped layers will now act as diffusion barriers preventing full oxidation of the layers by atmospheric oxygen.

\subsection{Secondary Ion Mass Spectroscopy (SIMS)}

In total 146 linescans were taken to sputter through two layers of the multilayer structure and obtain the oxygen data. The first 72 linescans corresponded to sputtering of the Sm doped layer, and the final 48 linescans to sputtering within the undoped $\mathrm{CeO}_{2}$ layer.

The final depth of the line scan trench was measured using optical interferometry and found to be $60 \mathrm{~nm}$ deep. The sputter rate was estimated at $0.017 \mathrm{nms}^{-1}$ (i.e. $0.42 \mathrm{~nm}$ per line scan cycle).

Therefore, using the sputter rate measurement and knowing the number of cycles for each layer, approximate layer thicknesses of $28 \mathrm{~nm}$ and $19 \mathrm{~nm}$ for the doped layer and the undoped layer respectively were obtained. The measurements correspond well to the expected layer thickness of $30 \mathrm{~nm}$ for the $\mathrm{Sm}$ doped layer and $20 \mathrm{~nm}$ for the $\mathrm{CeO}_{2}$ layer. We were thus, rather surprisingly, able to measure the lateral oxygen diffusion within the separate layers, a technique that will undoubtedly be very useful in a wide variety of samples of this type.

The ${ }^{18} \mathrm{O}$ and ${ }^{16} \mathrm{O}$ signals from each cycle corresponding to each layer were added, and then the ratio was taken to obtain the ${ }^{18} \mathrm{O}$ isotopic fraction diffusion profile in the separate layers. Figure 12 shows the ${ }^{18} \mathrm{O}$ diffusion profile in the $\mathrm{CeO}_{2}$ layer.

To obtain values for $\mathrm{D}$ and $\mathrm{k}$, the ${ }^{18} \mathrm{O}$ normalized isotopic diffusion profile, $\mathrm{C}^{\prime}(\mathrm{O})$, is fitted to the solution of Fick's $2^{\text {nd }}$ law of diffusion for a semi-infinite medium shown below in equation $1^{\text {[23] }}$ using a regression analysis fit in Matlab. ${ }^{[24]}$ 
$C^{\prime}(O)=\frac{C_{x}-C_{b g}}{C_{g}-C_{b g}}=\operatorname{erfc}\left(\frac{x}{2 \sqrt{D t}}\right)-\exp \left(h x+h^{2} D t\right) \operatorname{erfc}\left(\frac{x}{2 \sqrt{D t}}+h \sqrt{D t}\right)$

where, $\mathrm{h}=\frac{\mathrm{k}}{\mathrm{D}}, \mathrm{C}_{\mathrm{x}}$ is the ${ }^{18} \mathrm{O}$ isotopic fraction at depth $\mathrm{x}, \mathrm{C}_{\mathrm{g}}$ the isotopic fraction of ${ }^{18} \mathrm{O}$ in the gas phase and $\mathrm{C}_{\mathrm{bg}}$ the ${ }^{18} \mathrm{O}$ background isotopic fraction, and $\mathrm{t}$ the ${ }^{18} \mathrm{O}$ anneal time.

Figure 13 shows the ${ }^{18} \mathrm{O}$ isotopic fraction, $\mathrm{C}^{\prime}(\mathrm{O})$, diffusion profile for both the $\mathrm{CeO}_{2}$ and $\mathrm{Sm}$ doped $\mathrm{CeO}_{2}$ along with their calculated fits. In the case of the $\mathrm{Sm}$ doped $\mathrm{CeO}_{2}$ layer, the first few points of the linescan profile have not been included in the fit as they are adversely affected by sample edge effects. ${ }^{[11]}$ This makes estimating the surface exchange coefficient, $\mathrm{k}$, impossible however the diffusion coefficient, $\mathrm{D}$, can still be calculated. The values obtained for $\mathrm{D}$ in the multilayer structure were $2.8 \times 10^{-8} \mathrm{~cm}^{2} \mathrm{~s}^{-1}$ for the $\mathrm{Sm}$ doped $\mathrm{CeO}_{2}$ layer and $2.6 \times 10^{-8} \mathrm{~cm}^{2} \mathrm{~s}^{-1}$ for the $\mathrm{CeO}_{2}$ layer.

The values of $\mathrm{D}$ obtained for both the doped and undoped $\mathrm{CeO}_{2}$ layers are surprisingly similar. The value for the undoped $\mathrm{CeO}_{2}$ layer is much larger than expected for pure $\mathrm{CeO}_{2}$, however, this is only an 'apparent' value due to the manner in which the tracer oxygen diffuses through the multilayer. During the anneal experiment the oxygen tracer can diffuse very rapidly laterally along the Sm doped ceria layers. Because the layers are very thin, diffusion in the vertical direction within the doped layer is negligible with respect to the lateral diffusion length $(\sim 500 \mu \mathrm{m}$, Fig. 13$)$. Therefore, at the start of the anneal experiment the ${ }^{18} \mathrm{O}$ tracer diffuses rapidly in the $\mathrm{Sm}$ doped $\mathrm{CeO}_{2}$ layers, and more slowly in the $\mathrm{CeO}_{2}$ layers (Fig 14a). However, at the interface between the layers the tracer can diffuse from the doped to the undoped layer (Fig 14b). The undoped layers are only $20 \mathrm{~nm}$ thick therefore the distance needed for the tracer concentration to equalize in the layer from both directions is only $10 \mathrm{~nm}$. Under the annealing conditions used and an estimated D value of $10^{-11} \mathrm{~cm}^{2} \mathrm{~s}^{-1}$ for $\mathrm{CeO}_{2}{ }^{[25]}$ it would take only $0.001 \mathrm{~s}$ for this distance to be travelled; the total anneal time was 2250 seconds. Thus diffusion along the interface and into the undoped layer is dominating the tracer diffusion in this region. The final diffusion front formed after time $t$ is shown in Fig 14(c), and only an 'apparent' $\mathrm{D}$ value can be measured for the $\mathrm{CeO}_{2}$ layer. The situation may be analogous to a 
Harrison type A diffusion whereby diffusion in the grain boundaries is much faster than bulk diffusion in the grains, such that $\sqrt{ }(\mathrm{Dt})$ is much larger than $\mathrm{d}$ (where $\mathrm{d}$ is the grain size). Typically this is observed in polycrystalline materials with grain boundaries of the order of nanometres compared to the much larger micron sized grains of the bulk, and diffusion occurring in three dimensions. Although the aforementioned inequality is attained for diffusion in the $\mathrm{CeO}_{2}$ layer, and thus has similarities to Harrison type A diffusion, at the interface however, diffusion is two dimensional and the size of the interface relative to the thickness of the $\mathrm{CeO}_{2}$ are comparable.

Using the diffusion values obtained by the ion exchange method conductivities were calculated using the Nernst-Einstein equation. ${ }^{[26]}$ Values of $0.023 \mathrm{Scm}^{-1}$ for $\mathrm{CeO}_{2}$ and $0.024 \mathrm{Scm}^{-1}$ for the Sm doped $\mathrm{CeO}_{2}$ at $970 \mathrm{~K}$ were obtained. The values of oxygen diffusion coefficient found in the Sm doped layers correlate well with the conductivity data obtained from the 50 multilayer structure at $969 \mathrm{~K}$ which was measured as $0.0393 \mathrm{Scm}^{-1}$. The correlation between the electrical conductivities and the conductivities calculated using the ionic diffusion data indicate that the conduction method in the samples analyzed at $\sim 973 \mathrm{~K}\left(\right.$ i.e. $700^{\circ} \mathrm{C}$ ) is dominated by ionic conductivity.

The results presented here demonstrate a clear anomaly between the microstructure and the expected thin film conductivity. The conductivity has been measured to be similar to bulk samaria doped ceria and the oxygen diffusion experiment has supported the hypothesis that the measured conductivity is due to oxygen as the charge carrier. However, a significant fraction of the microstructure contains $\mathrm{Ce}(\mathrm{III})$ oxidation in both the as-grown and annealed samples. Moreover the overall conductivity is in agreement with bulk $\mathrm{Ce}_{0.85} \mathrm{Sm}_{0.15} \mathrm{O}_{2}$.

In order to resolve this apparent anomaly there are two key questions that must be answered; 1 . What is the role of the oxygen vacancies associated with the Ce(III)? 2. Why do we not observe significant electronic conductivity given the existence of the Ce(III)? We believe that two (possibly related) hypotheses that may answer question 1 . will also provide a solution to question 2 . If the $\mathrm{Ce}(\mathrm{III})$ is locally stabilised during growth in a specific region, then the associated oxygen vacancies may be 
immobile. This is possibly due to the stabilisation of a bixbyite or normally metastable fluorite structure within a localised region of the sample. Whilst this observation of stable $\mathrm{Ce}^{3+}$ content is surprising given the propensity for $\mathrm{Ce}(\mathrm{III})$ bulk oxides to rapidly oxidise to $\mathrm{Ce}(\mathrm{IV})$, it has also been demonstrated that on the nanoscale the stability of the $\mathrm{Ce}^{3+} / \mathrm{Ce}^{4+}$ states in $\mathrm{CeO}_{2}$ and doped $\mathrm{CeO}_{2}$ differs markedly from the bulk oxides. ${ }^{[27-29]}$ Indeed in nanoparticles of $\mathrm{CeO}_{2}$ a difference between the bulk and surface of a particle has been shown, with the bulk $7 \% \mathrm{Ce}^{3+}$ and the surface $83 \%$ $\mathrm{Ce}^{3+} \cdot{ }^{[28]}$. These measurements were made from nanoparticles and not from thin films, as is the case here, but there was a clear relationship between the size of the nanoparticles and the proportion of $\mathrm{Ce}^{3+}$ present. This may suggest that the films deposited are nucleating as sub $15 \mathrm{~nm}$ particles and it is this effect that causes growth of a layer with anomolous valence state. It is also proposed by Wu et $\mathrm{al}^{[27]}$ that the planes available for growth control the extent of reduction of the $\mathrm{CeO}_{2}$ layer, and in the case of constrained doped thin films this may be a key feature. Further it has been identified in $\mathrm{Tb}$ doped $\mathrm{CeO}_{2}$ that nanoparticulates with high $\mathrm{Ce}^{3+}$ concentration segregate, although the mechanism for this is unclear. ${ }^{[28]}$ Consequently it is conceivable that nanodomains with high $\mathrm{Ce}^{3+}$ concentration are segregating in the doped layer and the presence of the low angle grain boundaries may be preventing their oxidation. By locking the vacancies within these regions they will not contribute to the overall ionic conductivity. A normally meta stable structure may be stabilised during PLD growth, especially with the epitaxial layer by layer growth conditions. Moreover, the blocking of diffusion pathways may be the interface between $\sim \mathrm{Ce}_{2} \mathrm{O}_{3}$ and $\mathrm{CeO}_{2}$ regions. The processing steps and procedures associated with ac impedance spectroscopy and the ${ }^{18} \mathrm{O}$ exchange require that the sample is equilibrated with respect to oxygen. If the oxygen vacancies were fully mobile the multilayer would reach full oxidation, further evidence for the existence of "micro-domains." To answer the second question we may use a similar argument. The micro structural characterization shows variations along, and across, the multilayers with abrupt changes in $4^{+} / 3^{+}$ratio and the 
atomically sharp doped/undoped interface. If the micro-domains are discrete regions with immobile charge carriers there may be no continuous pathway for electronic conductivity.

\section{Conclusions}

This multilayer structure has clearly developed a more complicated chemical microstructure than the original growth would expect. There must be mixed valence cerium present (in discrete regions/domains), and this is seen in the EELS. There is a spatial variation in the Ce valence and this has been mapped and there is an overall difference between the doped and undoped layers. This variation remains after annealing to a lesser extent (almost within noise).

We are led to the conclusion that, while the multilayer structure can be grown successfully with a sharp doped/undoped interface characteristic, there exist discrete micro-domains that do not contribute to the overall conductivity, both electronic and ionic. What remains is the continuous pathway for oxygen ion conduction that is of similar magnitude to bulk SDC.

\section{Experimental}

\subsection{Sample Synthesis}

Samples were grown by pulsed laser deposition (PLD) at the Center for Nanophase Materials Sciences at Oak Ridge National Laboratory. MgO (001) substrates (Coating and Crystal Technology, US) were selected for quality by visual inspection and x-ray diffraction (PANalytical X'Pert Pro MRD) using orthogonal rocking curve analysis. Substrates with multiple peaks were discarded, with the remaining substrates ranked in order of peak width (FWHM) and symmetry. Surface quality was evaluated using AFM (Dimension 3100) to ensure < $2 \mathrm{~nm}$ overall roughness. Substrates were 
cleaned with acetone, dipped in phosphoric acid (85\%) for 5 seconds, washed with DI water, and blown dry with $\mathrm{N}_{2}$ prior to mounting in the PLD chamber.

PLD targets of $\mathrm{CeO}_{2}$ and 15 at $\% \mathrm{Sm}$-doped $\mathrm{CeO}_{2}$ (SDC) were sintered using commercially available high purity powders (Alfa Aesar). The powders were ball milled using an agate ball mill until all agglomerates were removed, uni-axially pressed into green bodies, and then sintered at $\sim 1500^{\circ} \mathrm{C}$ for 20 hours. The sintered targets were checked for compositional homogeneity by x-ray fluorescence (XRF) mapping (Shimadzu $\mu$ XRF 3100) and the composition was checked around the area that is used for ablation target.

The PLD procedure involved growth at approximately $1 \AA$ per shot at $5 \mathrm{~Hz}$ and with the $\mathrm{MgO}$ substrate mounted to a heating stage typically held at $800^{\circ} \mathrm{C}$ and $5 \mathrm{~cm}$ from the ablation target. Deposition was carried out at $\sim 2.4 \mathrm{Jcm}^{-2}$ in 10 mtorr of UHP oxygen. Based on independent calibrations using a thermocouple attached to an $\mathrm{MgO}$ substrate, the actual (clip mounted) sample temperature was found to be $\sim 475^{\circ} \mathrm{C}$.

Although no RHEED oscillations were observed during growth, the substrate pattern streaked out after approximately 500 shot, and remained streaky until the end of growth, suggesting that high quality layered structures had formed in all cases examined here. The final films were also measured using XRD, and all exhibited cube-on-cube epitaxial relationship. A set of samples was grown, including a 50 cycle multilayer (of $20 \mathrm{~nm} \mathrm{CeO}_{2}, 30 \mathrm{~nm} \mathrm{Ce}_{0.85} \mathrm{Sm}_{0.15} \mathrm{O}_{2-\mathrm{x}}$ ) and a 400 cycle multilayer of the same composition. The choice of layer thicknesses were the result of observing how much material was required to transition from the substrate pattern to a streaky film pattern, which in this case turned out to be $\sim 50 \mathrm{~nm}$. Each sample contained such a buffer layer, which allowed the $\mathrm{MgO} /$ ceria mismatch to be relieved, and subsequent epitaxial growth through the multilayers to proceed with reasonably high quality. For isotope exchange experiments, the samples were capped with an $\mathrm{MgO}$ layer of $~ 100 \mathrm{~nm}$ using electron beam deposition. 
Here, we report microstructural conclusions representative of all the samples grown. Specific EELS experiments were conducted on an as-grown sample and further samples were annealed in air with an initial ramp rate of $10^{\circ} \mathrm{C} / \mathrm{min}$ to $400^{\circ} \mathrm{C}$, then at a rate of $\sim 1^{\circ} \mathrm{C} / \mathrm{min}$ up to the peak temperature of $\sim 800^{\circ} \mathrm{C}$ where the sample was held for 2 hours.

\subsection{Conductivity Measurement}

Conductivity measurements were acquired by AC impedance spectroscopy using a Solartron 1260 frequency response analyzer with a Solartron 1296 dielectric interface. The experiments were performed using a two electrode configuration and an AC amplitude of $1 \mathrm{~V}$ with a logarithmic sweep over the frequency range of $13 \mathrm{MHz}$ to $1 \mathrm{~Hz}$ at $50^{\circ} \mathrm{C}$ increments between $400-800^{\circ} \mathrm{C}$. A voltage amplitude of $1 \mathrm{~V}$ was selected in order to reduce data scatter due to noise. The linearity in the current response was confirmed by checking that the spectra observed were not dependant on the amplitude over a range of $10 \mathrm{mV}$ to $1 \mathrm{~V}$. Silver electrodes were painted onto freshly exposed edges of the samples and silver leads were attached in the configuration shown in Fig. 1(b). The whole assemblies were then sintered at $700^{\circ} \mathrm{C}$ for 2 hours to ensure good contact between the silver electrodes and the multilayers. Optical and scanning electron microscopy (FEI Phenom) was used to check the adhesion of the silver to the multilayers to ensure optimal electrical contact. The geometry shown in Fig. 1(b) was found to achieve the best overall contact and mechanical stability. Measured impedance spectra were fitted using equivalent circuit modelling in Zview. ${ }^{[30]}$

\subsection{Electron microscopy imaging and analysis}

All transmission electron microscopy results were obtained using two scanning transmission electron microscopes (STEM, FEI Titan 80-300). Both systems are fitted with a monochromator and electron 
energy loss spectroscopy (EELS) system (Gatan Tridiem) to achieve an energy resolution better than $0.2 \mathrm{eV}$. One system is fitted with a Cs-corrector on the imaging lens (Imperial College London), while the other has a corrector on the probe-forming lens (Ohio State University). Sample preparation was achieved using both conventional cross sectional preparation and focussed ion beam preparation. The FIB preparation was carried out at $5 \mathrm{kV}$ or $2 \mathrm{kV}$ to minimise $\mathrm{Ga}$ implantation and amorphisation. The conventional preparation route followed the standard procedure for cross sectional TEM sample preparation (as described elsewhere ${ }^{[31]}$ ). All samples were polished using low energy argon ion milling (PIPS, Gatan or Nanomill, Fischione) and were plasma cleaned prior to insertion in the STEM.

\subsection{SIMS}

To measure the diffusion, $\mathrm{D}$, and surface exchange, $\mathrm{k}$, coefficients of a 50 multilayer $\mathrm{Ce}_{0.85} \mathrm{Sm}_{0.15} \mathrm{O}_{2} / \mathrm{CeO}_{2}$ sample, ion exchange and SIMS linescanning were carried out. In order to limit the diffusion to the lateral direction during the isotopic exchange step, a $100 \mathrm{~nm}$ thick $\mathrm{MgO}$ blocking layer was deposited onto the sample surface as mentioned above. The sides of the sample were left exposed to allow diffusion along the deposited layers. The samples were initially annealed in an ${ }^{16} \mathrm{O}$ atmosphere at $970 \mathrm{~K}$ for several hours to ensure chemical equilibrium of the sample. The atmosphere was then enriched with $36 \%{ }^{18} \mathrm{O}$ and the sample ion exchanged at $970 \mathrm{~K}$ for approximately 20 minutes.

SIMS measurements were carried out using an Atomika 4500 SIMS instrument. The primary ion beam used was $1 \mathrm{keV} \mathrm{Cs}^{+}$with a beam current of $\sim 20 \mathrm{nA}$. The ${ }^{18} \mathrm{O}$ diffusion profiles were measured via SIMS linescanning. Initially, a large region of the sample was sputtered, using a $1 \mathrm{keV}$ Cs beam at $45^{\circ}$ to the surface to remove the $\mathrm{MgO}$ layer. In this case, both $\mathrm{Mg}$ and Ce signals were monitored. Removal of the $\mathrm{MgO}$ was stopped when the $\mathrm{Mg}^{+}$signal had reached $50 \%$ of its maximum bulk value. After removal of the $\mathrm{MgO}$, the samples were then repeatedly linescanned to obtain the ${ }^{16} \mathrm{O}$ and ${ }^{18} \mathrm{O}$ 
diffusion profiles in each of the layers. Along with ${ }^{16} \mathrm{O}^{-}$and ${ }^{18} \mathrm{O}^{-},{ }^{140} \mathrm{Ce}^{-},{ }^{156} \mathrm{CeO}^{-}$and ${ }^{168} \mathrm{SmO}^{-}$were also collected so changes in either the Ce or Sm could identify the different layers being sputtered. The sample was linescanned over a length of $1000 \mu \mathrm{m}$ (256 data points) and each secondary ion signal was collected for $5 \mathrm{~s}$. Each cycle taking $25 \mathrm{~s}$ to complete, and in total, 146 cycles were carried out. The final depth of the line scan trench, which was used to estimate sputter rates, was measured using optical interferometry.

\section{Acknowledgements}

The authors would like to thank Igor Kosacki for helpful discussions. We would like to acknowledge Paul Thomas (Gatan inc.) for invaluable advice. Funding was obtained by DOE access grant number 2009-022, the EPSRC (grant numbers EP/C51596X/1 and EP/D068924/1), DTA, The Royal Society, The Royal Academy of Engineering and the AtlantICC alliance partnership. Research conducted at the Center for Nanophase Materials Sciences and the Shared Research Equipment user facility was performed at Oak Ridge National Laboratory, and sponsored by the Division of Scientific User Facilities, U.S. Department of Energy.

Received: ((will be filled in by the editorial staff))

Revised: ((will be filled in by the editorial staff)) Published online: ((will be filled in by the editorial staff)) 
(a)

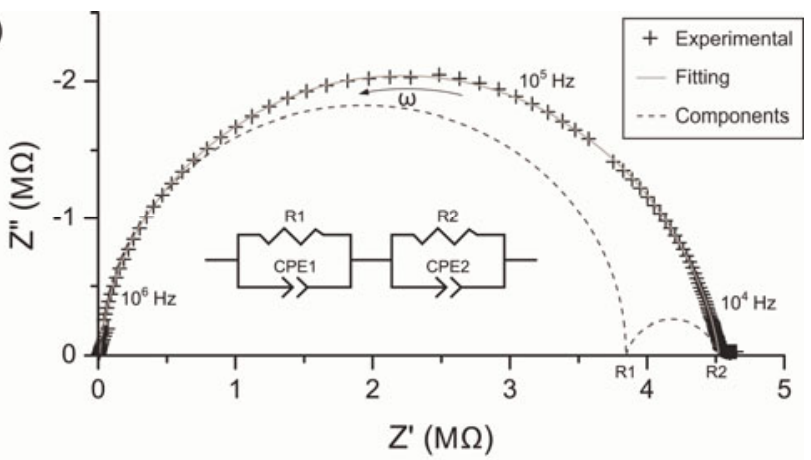

(b)

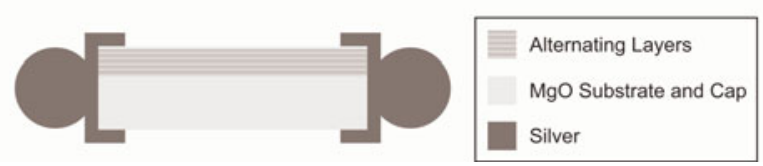

Figure 1. (a) A typical Nyquist plot of the impedance of the multilayer systems, 50 multilayer sample recorded at $400^{\circ} \mathrm{C}$ in static air together with the equivalent circuit (inset) used in the fitting. (b) The electrode geometry used for all measurements.

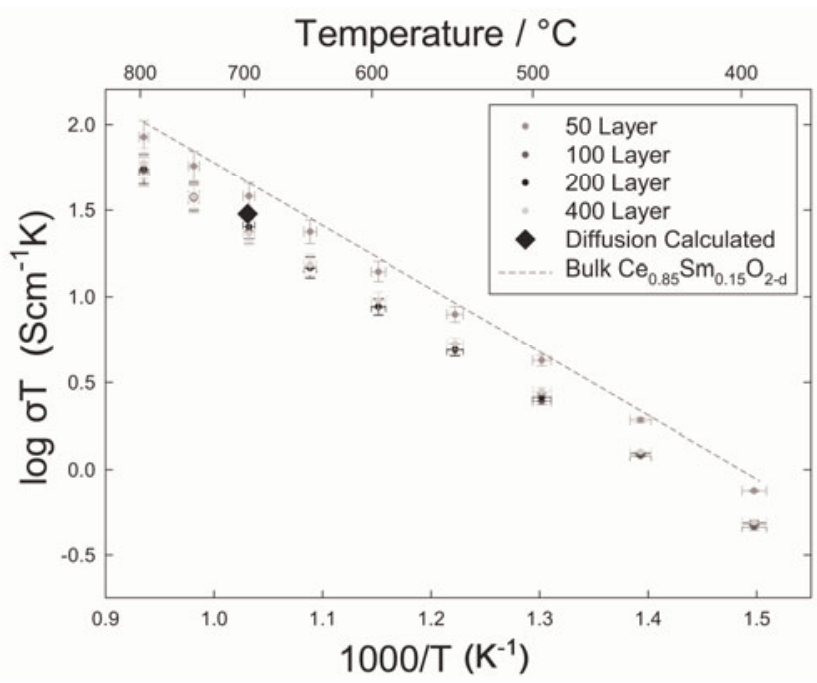

Figure 2. Temperature dependence of electrical conductivity of $\mathrm{CeO}_{2} / \mathrm{Ce}_{0.85} \mathrm{Sm}_{0.15} \mathrm{O}_{2-\mathrm{x}}$ multilayer systems with different number $n$ of layers where $n=50,100,200$ and 400.100, 200 and 400 layer samples show a great degree of overlap. Oxygen ion conductivity in a 50 layer sample, calculated from ion exchange experiments discussed later is also plotted along with bulk conductivity of $\mathrm{Ce}_{0.85} \mathrm{Sm}_{0.15} \mathrm{O}_{2-\mathrm{x} .}{ }^{[19]}$ This shows that the conductivity in this sample is dominated by the ionic species and does not differ greatly from bulk behavior. 


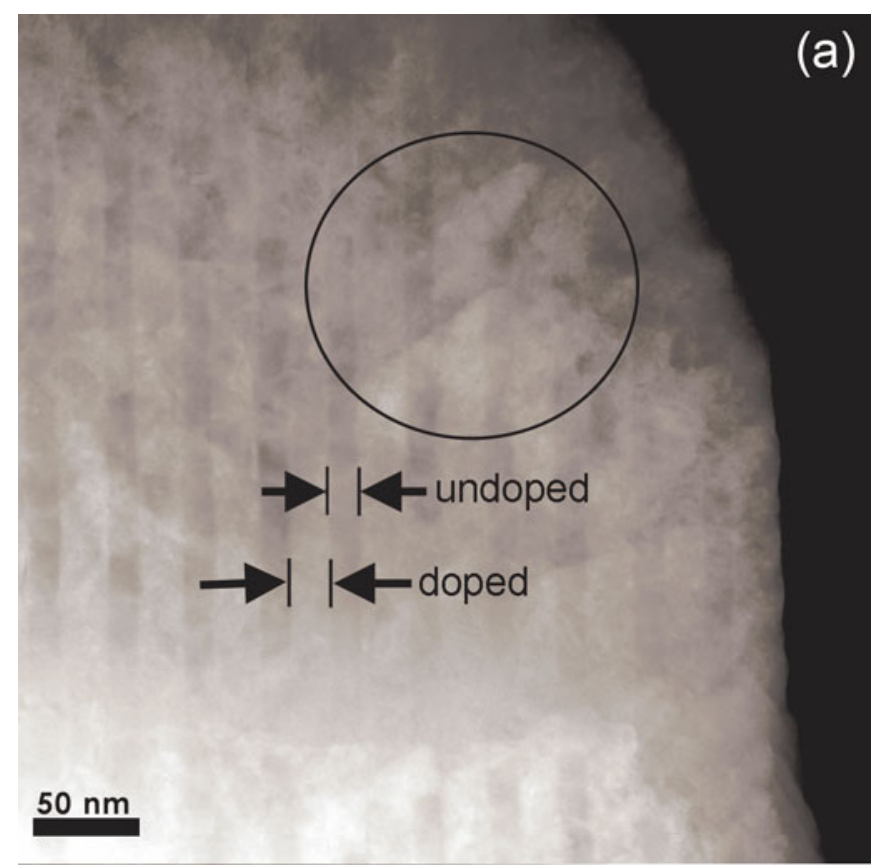

(b) Figure 3. Parallel acquisition HAADF (a) and bright field (b) STEM images of the multilayer structure. The growth direction is from left to right with undoped and doped layers marked and contrast features (lines running $\sim$ parallel with growth direction) from the same region of sample indicated in both images. (c) HRSTEM image showing good epitaxial growth. The growth direction is from left to right and the interface between doped and undoped is approximately down the centre of the image (marked with dotted line). 


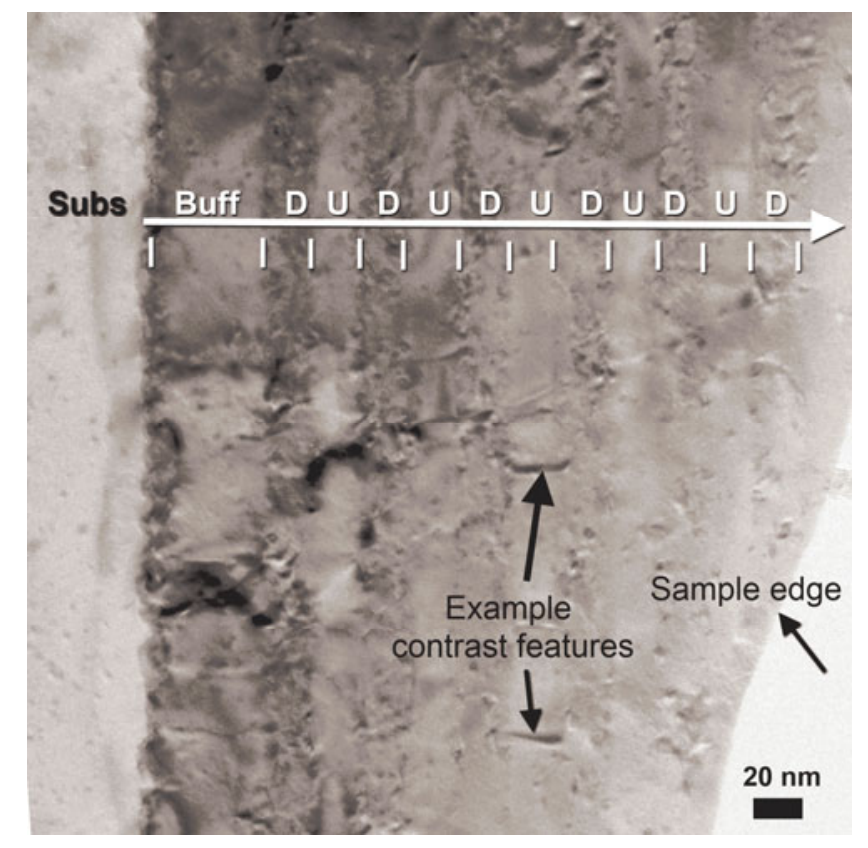

Figure 4. TEM image of multilayer structure with growth direction and approximate interface positions marked in white. $\mathrm{D}=$ doped layer and $\mathrm{U}=$ undoped. The edge of the sample is marked to indicate the lack of an amorphous layer and specific contrast features running through an undoped layer are also marked. 


\section{FUNCTIONAL Submitted to 1 ATER ALS}

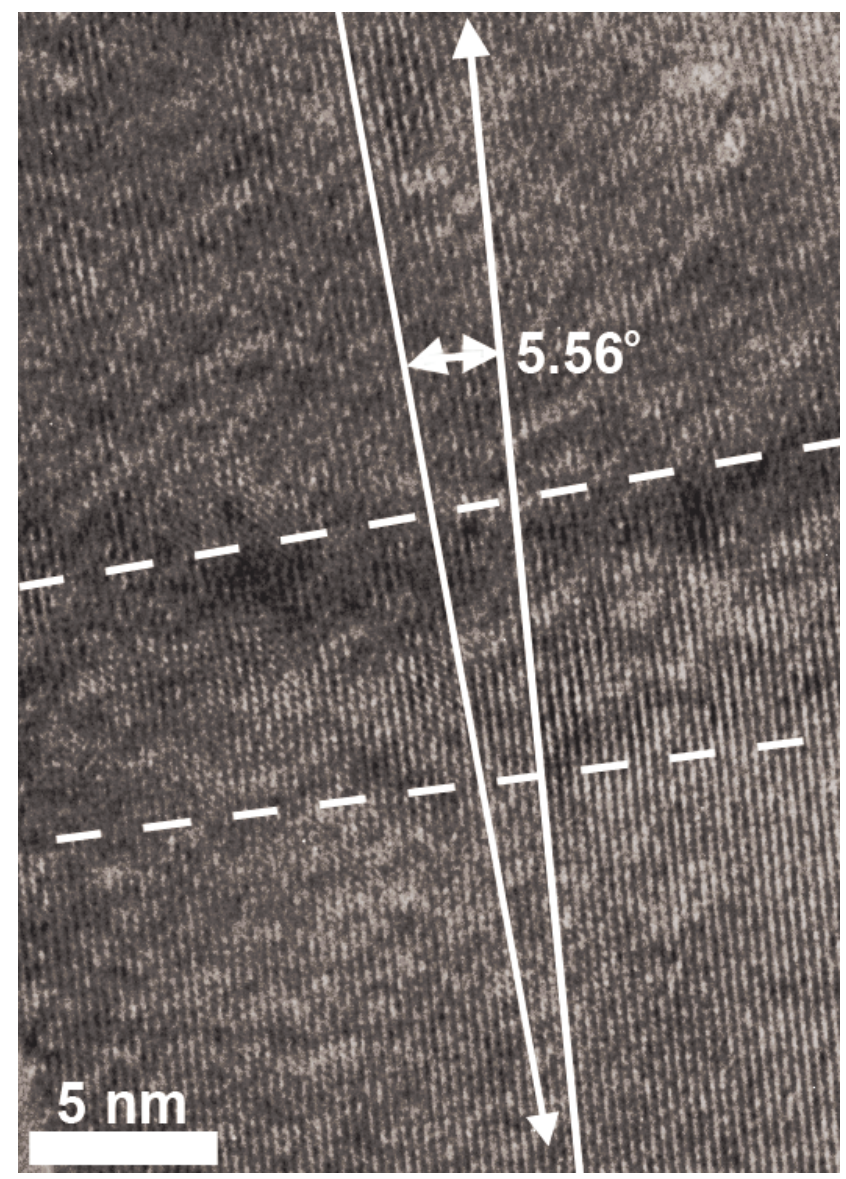

Figure 5. HRTEM image of a typical contrast feature, in this case a low angle grain boundary, from the as-grown sample. The multilayer growth direction is from right to left with the grain boundary running approximately parallel with the growth direction. The boundary is tilted and the approximate boundary region is marked by the dotted lines. 


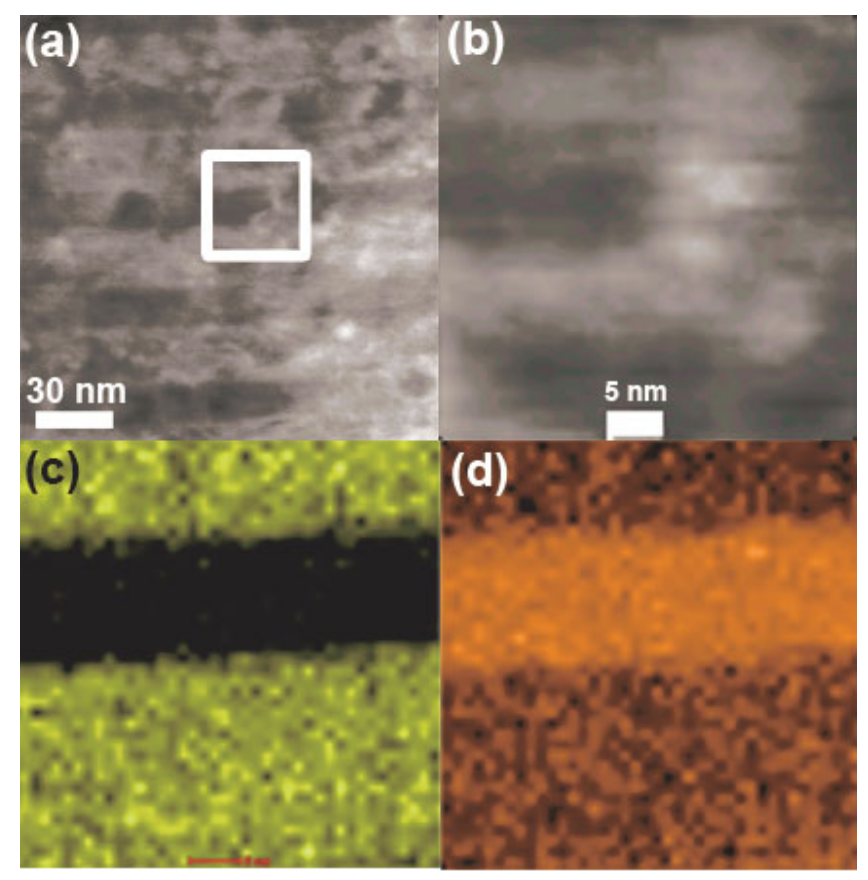

Submitted to

Figure 6. Energy dispersive x-ray spectroscopy maps over a small region of an as-grown

sample as marked in (a). (b) shows the STEM intensity with a contrast feature visible. (c) and (d) are the $\mathrm{Sm} \mathrm{K} \mathrm{K}_{\alpha}$ and $\mathrm{Ce} \mathrm{K}_{\alpha}$ signals respectively. 


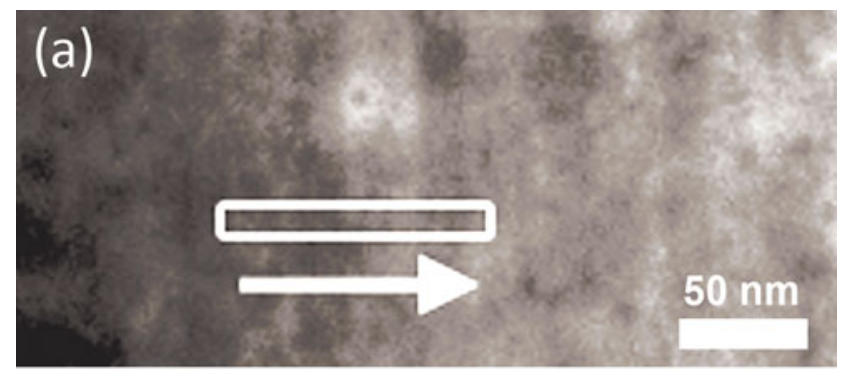

Submitted to

\section{ADNANEED
FUNCIONALL

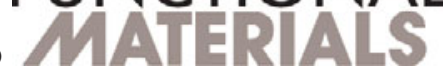

(b)

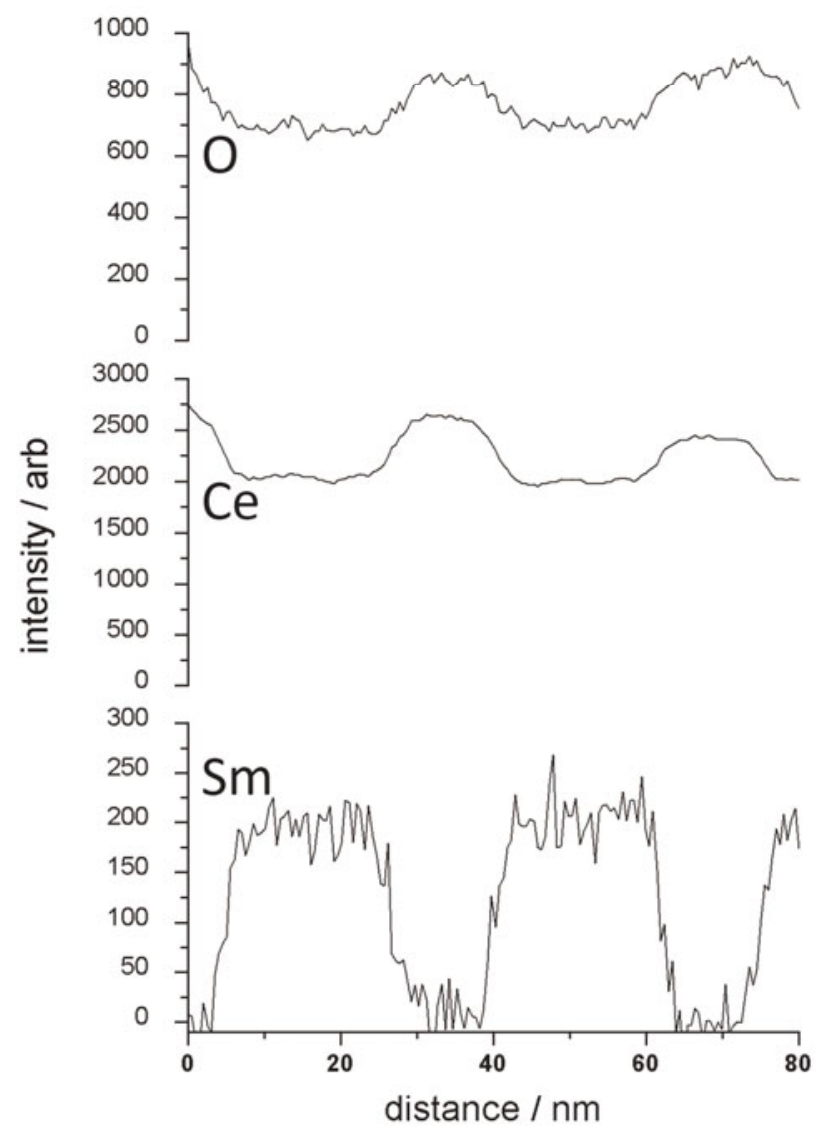

Figure 7. a) HAADF STEM image of as-grown multilayer with the line profile position marked. b) Cerium $\mathrm{M}_{4,5}$ edge extracted profile, oxygen $\mathrm{K}$ edge extracted profile and samarium $\mathrm{M}_{4,5}$ edge extracted profile showing the compositional homogeneity of the layers. 


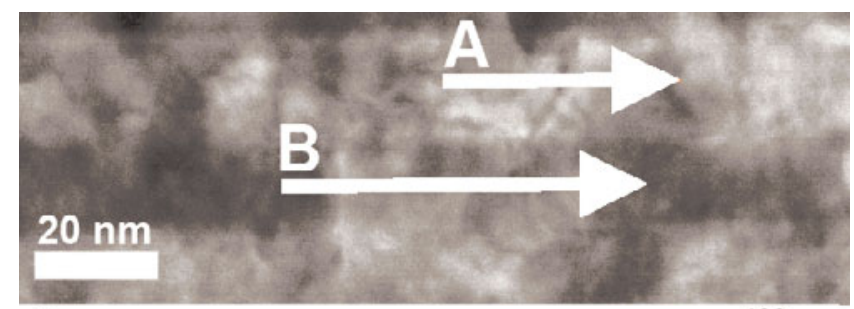

Submitted to 1 A
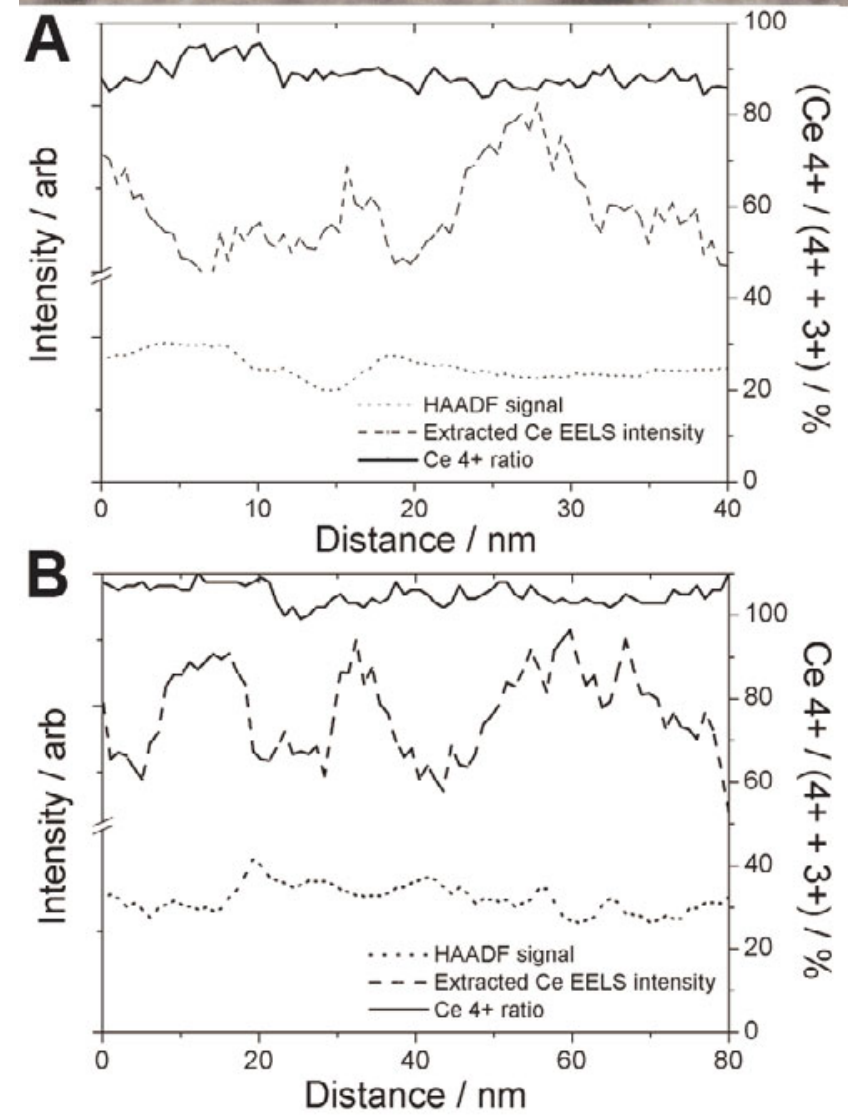

Figure 8. HAADF STEM image of as-grown multilayers with two profile positions marked, $\mathrm{A}=$ doped $\& \mathrm{~B}=$ undoped. Graphs show the HAADF signal intensity, plotted with the EELS Ce intensity profile. The MLLS profile (contribution from the $\mathrm{Ce}^{4+}$ ) is included on the $2^{\text {nd }} \mathrm{y}$ axis. 


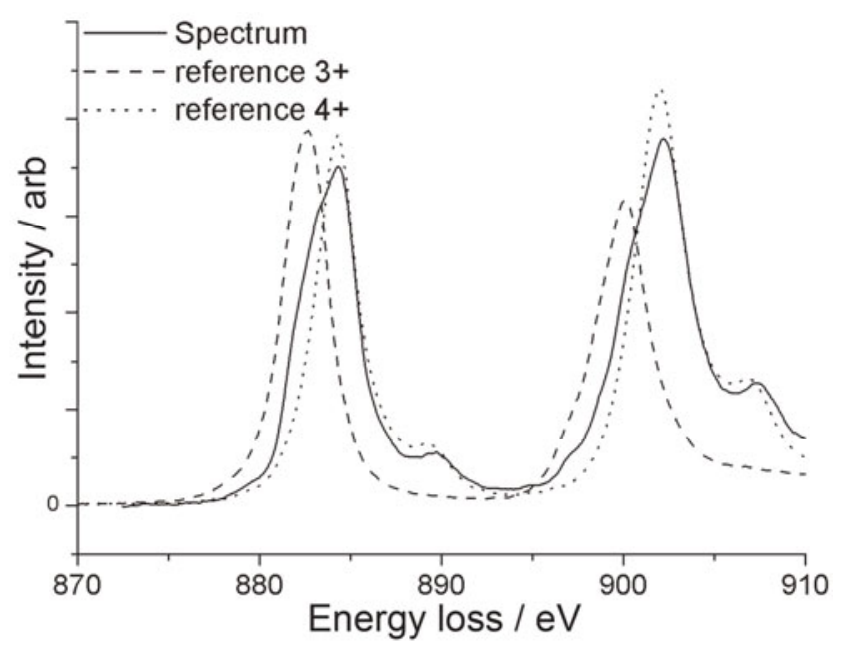

Submitted to FUNCTIONAL 14A

Figure 9. Example of MLLS fitting procedure. The two reference spectra are shown along with an experimental spectrum from the as-grown sample, within the undoped layer of the line profile. The energy window used for the fitting procedure was $875-895 \mathrm{eV}$. 


\section{Submitted to \\ ADYNANER
FUNCTONAL \\ 14 A E R A}
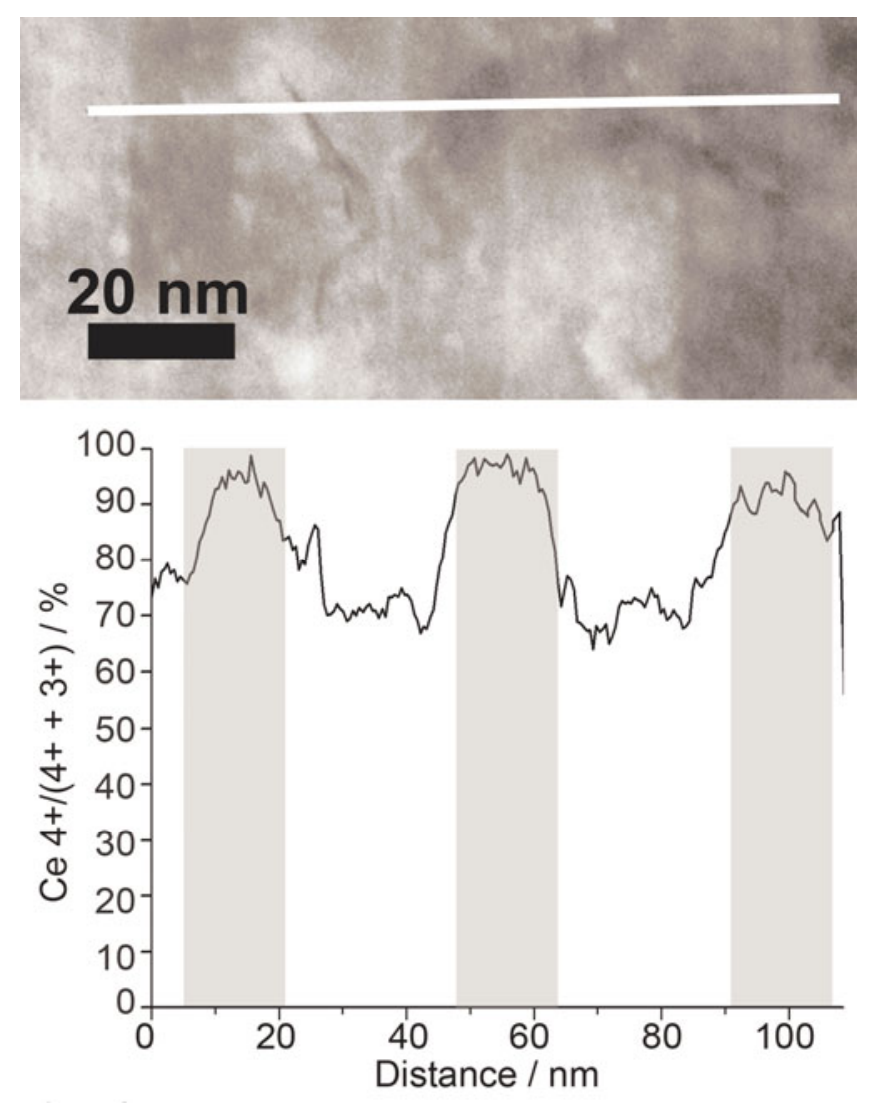

Figure 10. HAADF STEM image of the as-grown multilayers with line profile region marked with the 4+ / 3+ compositional variation extracted from MLLS fitting of the EEL spectra at each point on the line profile shown below. The approximate position of the undoped layer is marked in gray in the profile. 


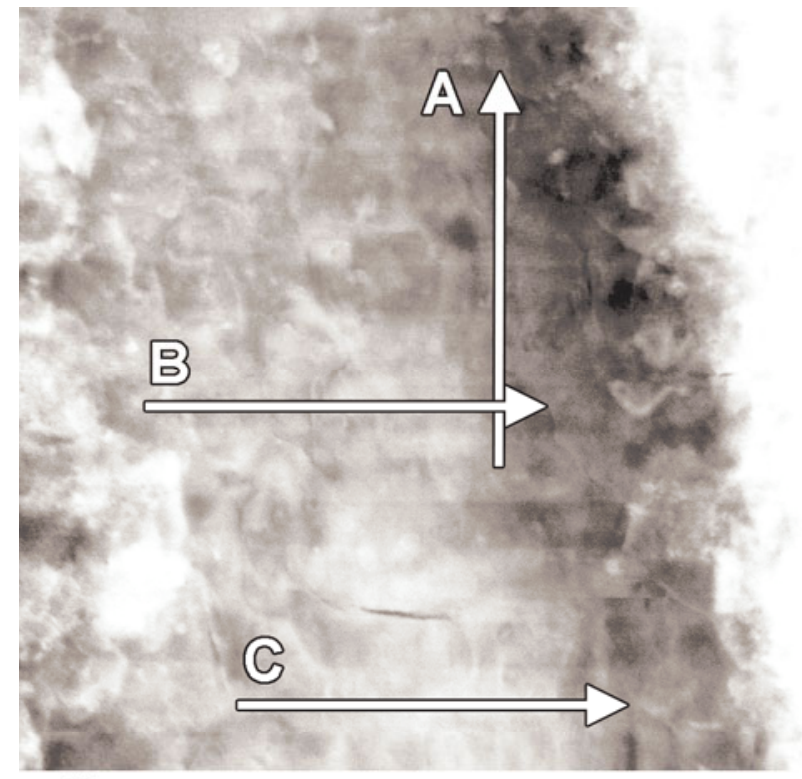

Submitted to

\section{FUNCTIONAL \\ 14ATEIAS}
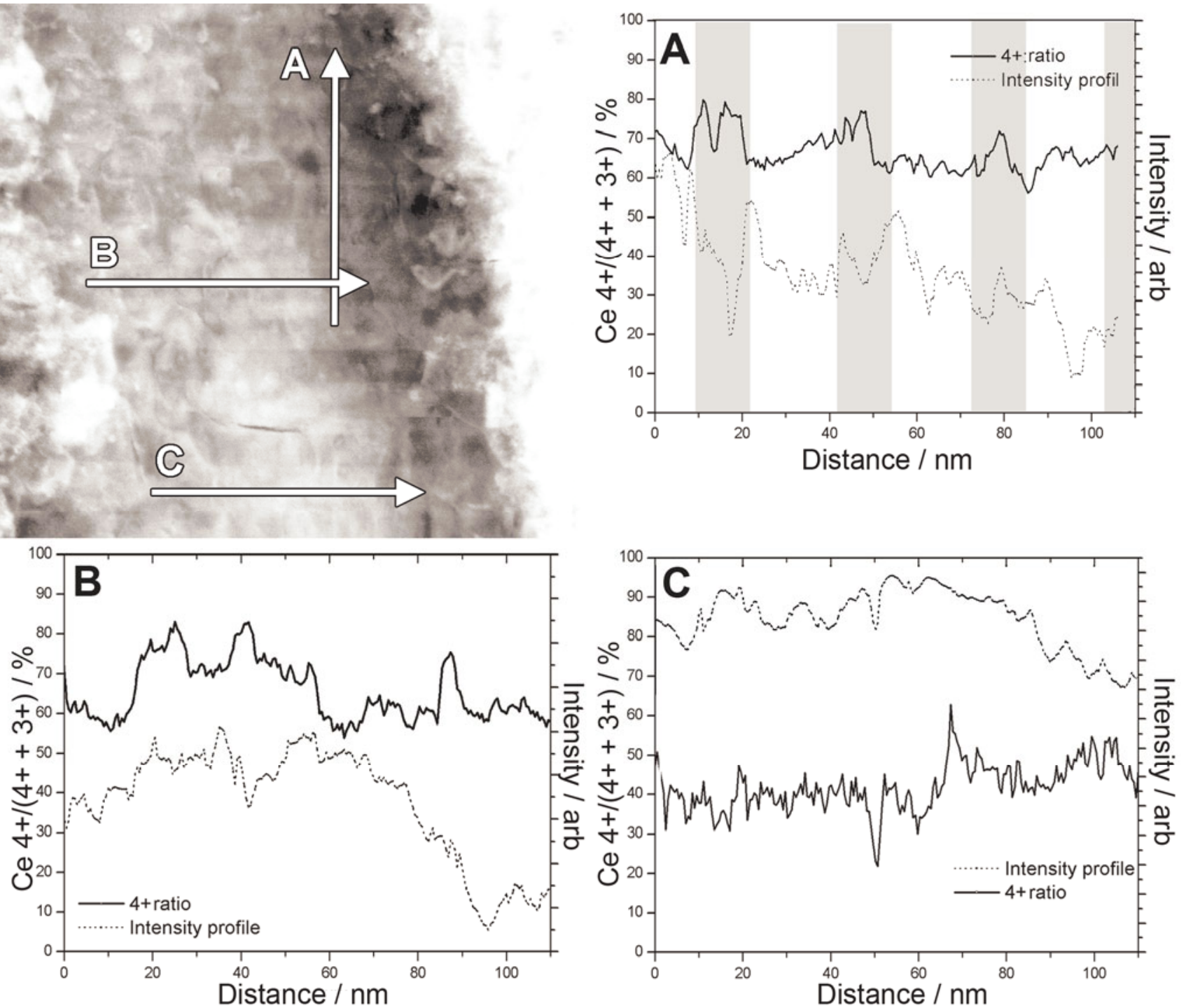

Figure 11. HAADF STEM image of annealed sample with profile positions marked; A -

Across multilayers, $\mathrm{B}$ - through the undoped layer, $\mathrm{C}$ - through the doped layer.

Accompanying graphs show ratio of valence states from MLLS fitting plotted against the

HAADF signal with the graph from (A) indicating undoped layers with the gray coloration. 

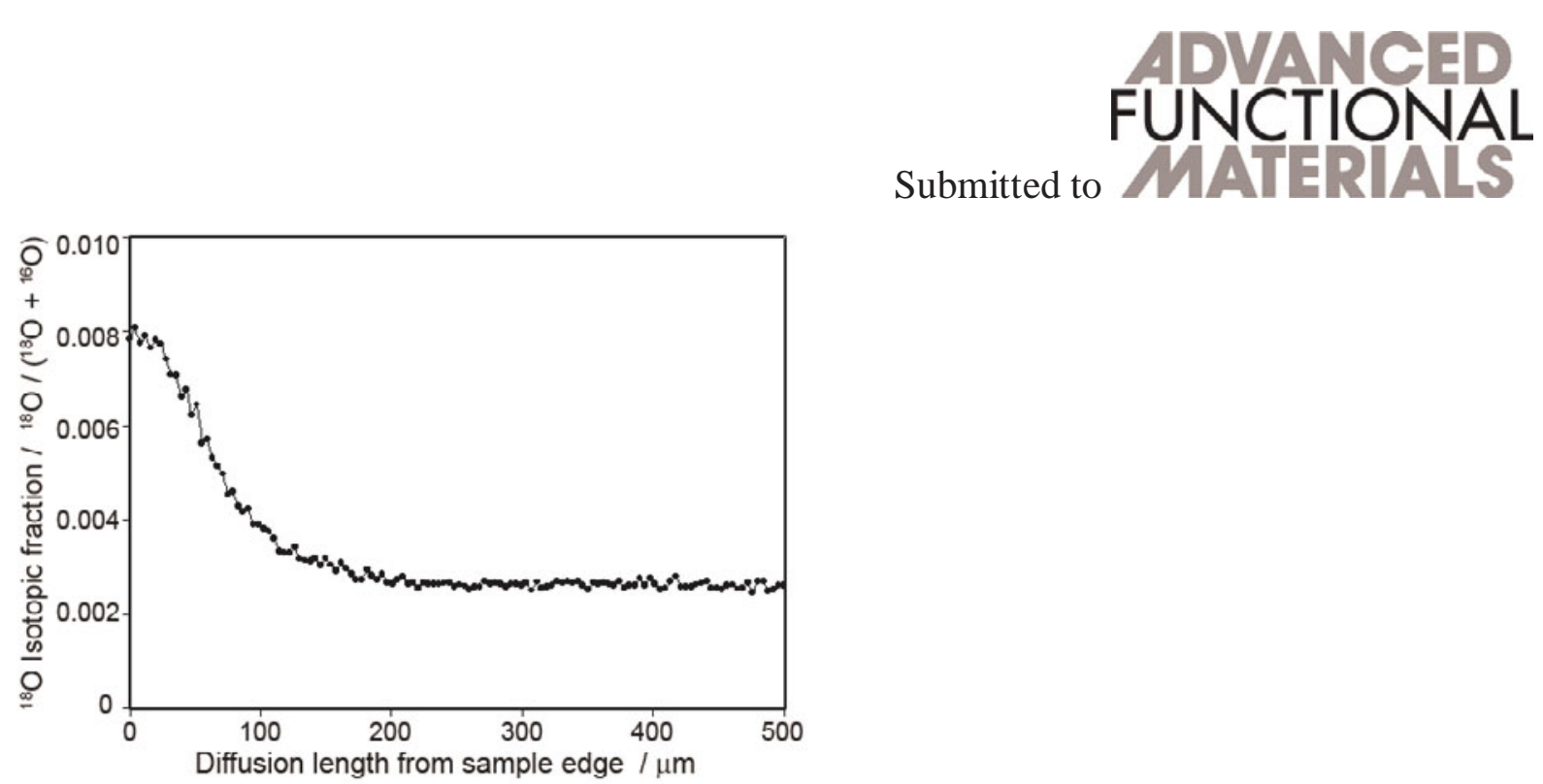

Figure 12. ${ }^{18} \mathrm{O}$ diffusion profile for the $\mathrm{CeO}_{2}$ layer.

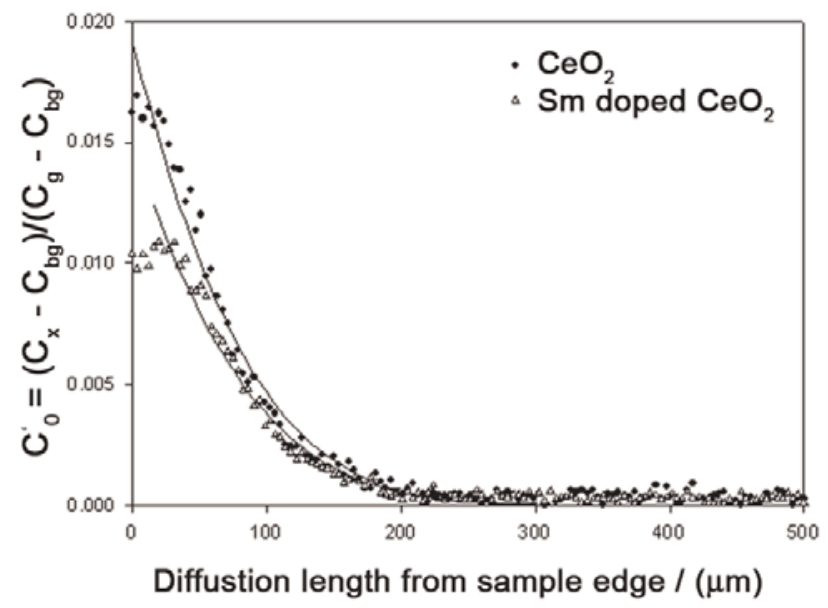

Figure 13. The normalized ${ }^{18} \mathrm{O}$ diffusion profile, $\mathrm{C}^{\prime}(\mathrm{O})$, for both the $\mathrm{CeO}_{2}$ and $\mathrm{Sm}$ doped $\mathrm{CeO}_{2}$ layer along with their calculated fits 


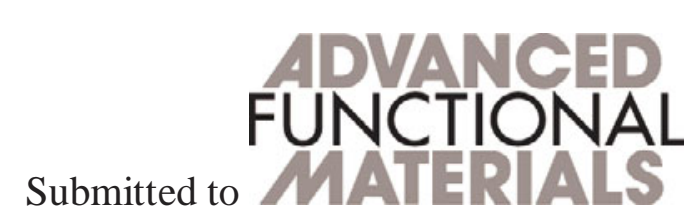

(a)

180

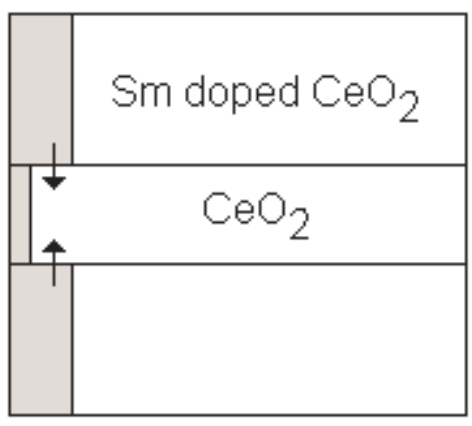

(b)

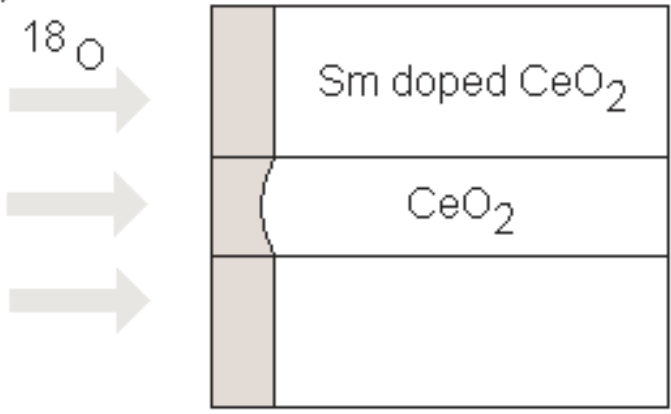

(c)

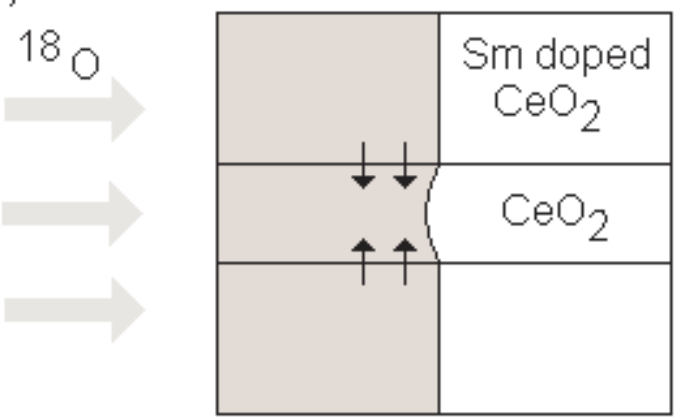

Figure 14. Schematic representation of the oxygen diffusion mechanism through the multilayer system during an ${ }^{18} \mathrm{O}$ tracer experiment 


\section{Supplementary information}

\section{Conductivity:}

Resistance values $(R)$ taken from impedance measurements were converted to sample dimension independent values of conductivity using equation SE1 where $h$ is the height of the samarium doped layers and $n$ is the total number of layer repeats. Further dimensions are illustrated in figure S1.

$\sigma=l / R w\left(h *_{n}\right)$

Conductivity measurements of undoped ceria grown on a magnesia substrate in an identical manner to those discussed here exhibited significantly higher resistance and therefore the contribution to the conduction of both the magnesia substrate and undoped ceria layers were assumed to be negligible.
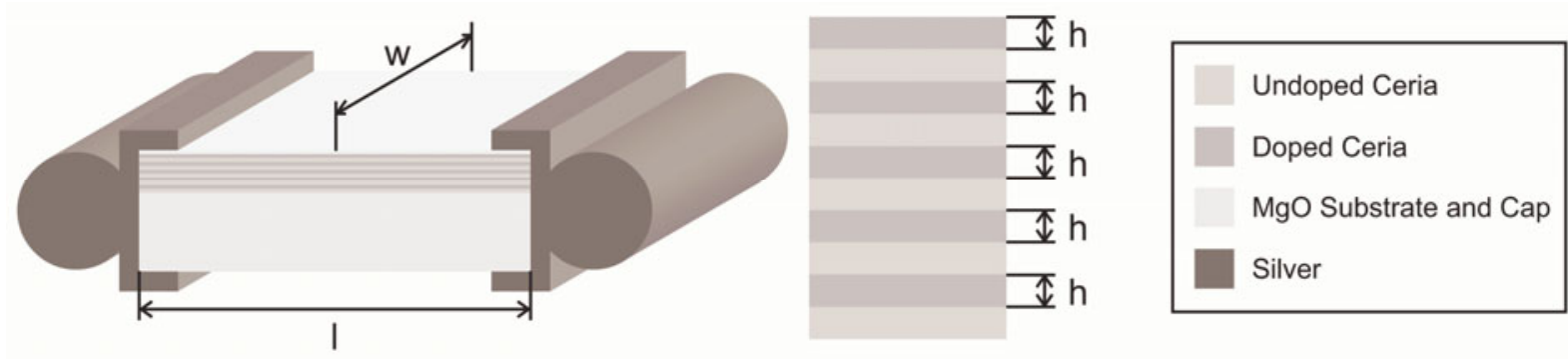

Figure S1: Schematic of the sample including measurement dimensions.

\section{EELS:}

The example spectrum shown in Fig. S2 shows the oxygen, cerium and samarium edges [used for the experiment] superimposed on the decreasing background associated with excitations at lower energy-losses. The background can be fitted to a power law function and before each ionisation edge, extrapolated under the edge and subtracted. Once this is completed the 
Submitted to

intensity in an energy window on each edge is integrated. Applying this procedure to each component in a spectrum image allows compositional profiles to be extracted.

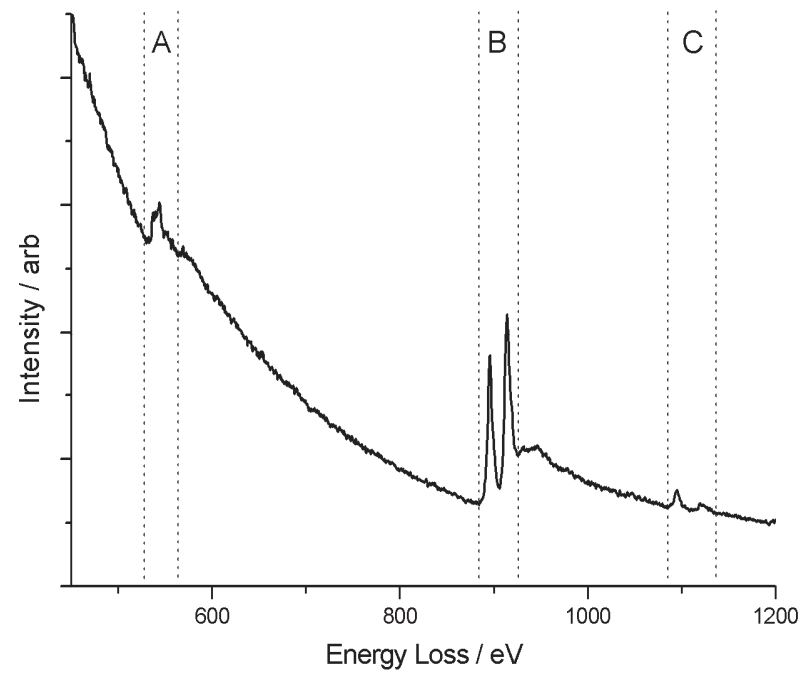

Figure S2. Example raw EEL spectrum from multilayer sample (...doped region) showing the energy windows used to extract the compositional profiles; A: Oxygen K-edge, B: cerium $\mathrm{M}_{4,5}$-edge, $\mathrm{C}$ : samarium $\mathrm{M}_{4,5}$-edge.

Each interface exhibits some roughness with the extent most pronounced at the substrate/buffer interface due to the large lattice mismatch between $\mathrm{MgO}(4.213 \AA)$ and $\mathrm{CeO}_{2}$ (5.411 $\AA$ ). Since the RHEED, XRD and TEM all imply an orientation relationship of (100) $\mathrm{MgO} / /(100) \mathrm{CeO}_{2}$ and $(020) \mathrm{MgO} / /(020) \mathrm{CeO}_{2}$ the elastic strain would suggest that an array of dislocations would form at the substrate/buffer interface.

To ensure the crystallography of the (100) $\mathrm{MgO}$ substrates, XRD scans were taken over the 2theta range of 22 degrees on the substrates at orientations of 0 and 90 degrees to confirm the presence of the 100 peak and absence of other peaks i.e. crystallographic planes 


\section{Submitted to FUNCTIONAL}

XRD Phi and Omega scans of the final films show that the films are aligned well to the substrate and that crystalline growth has been achieved
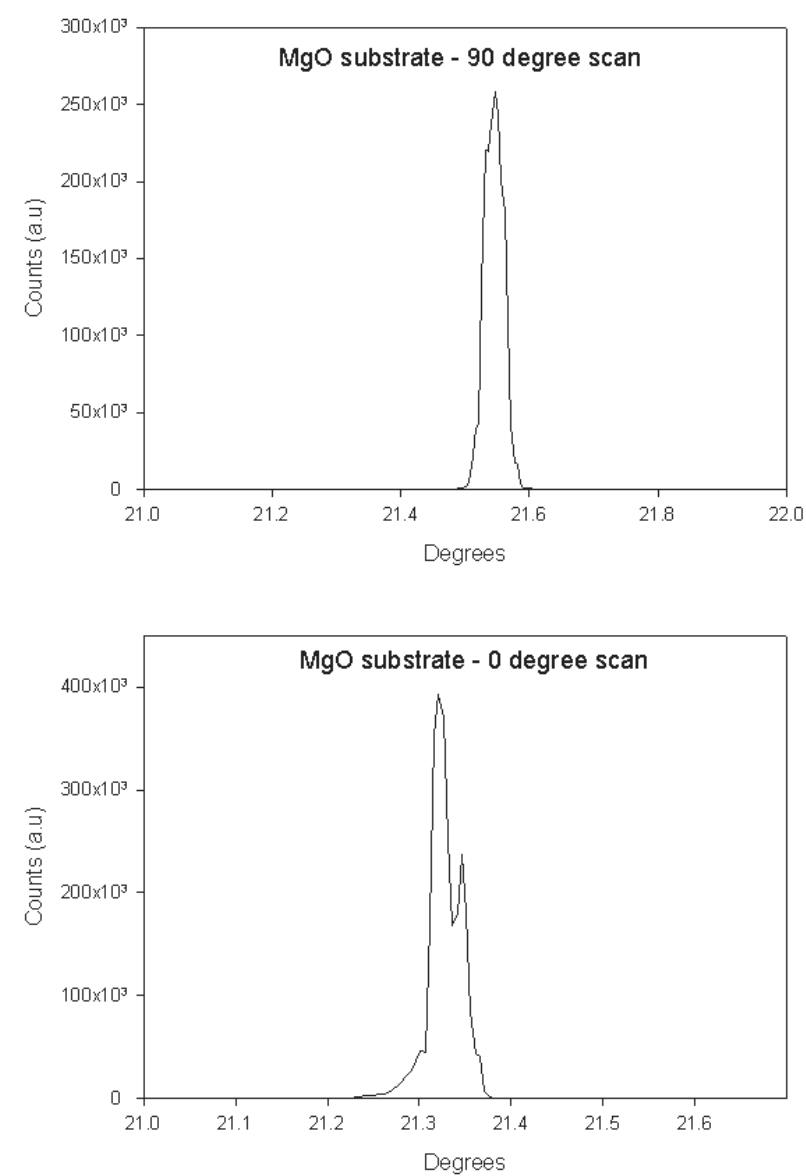

Figure S3: Example XRD scans from the $\mathrm{MgO}$ substrate.

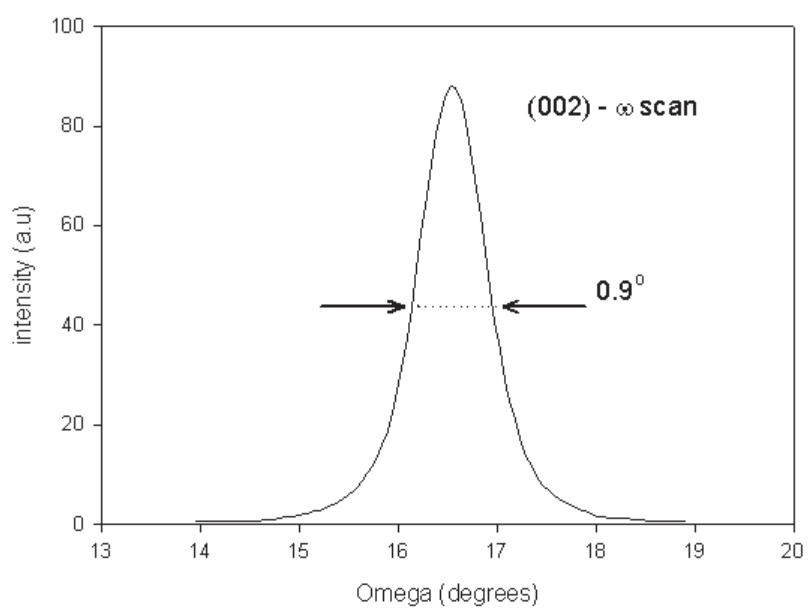


Submitted to

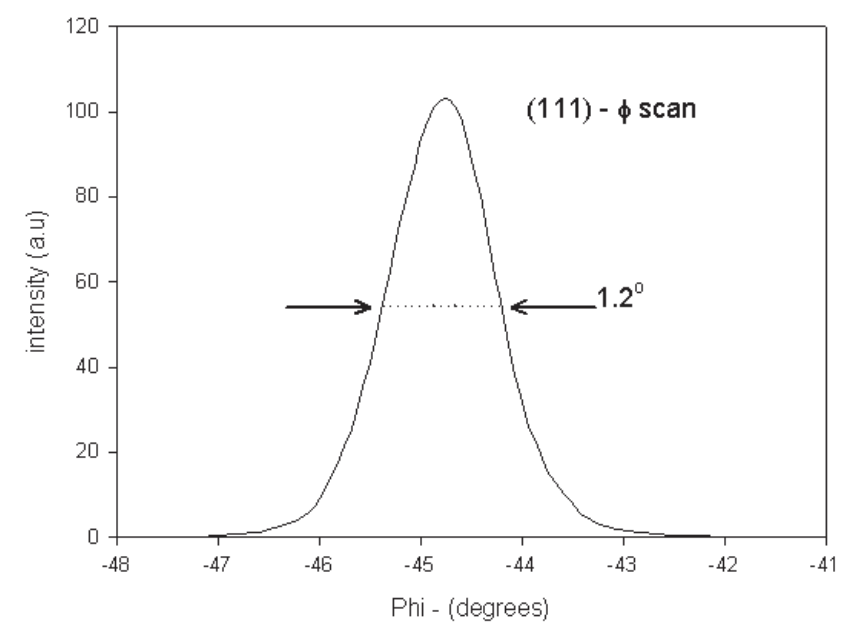

Figure S4: Example Phi and Omega scans from the as-grown (pre-capped) thin films.

References

[1] Kilner, J. A., Nature Materials, 2008, 7, 838-839.

[2] Kosacki, I., Rouleau, C. M., Becher, P. F., Bentley, J., Lowndes, D. H., Solid State Ionics, 2005, 176, 1319-1326.

[3] Goodenough, J. B., Nature, 2000, 404, 821-823.

[4] Kilner, J. A., Chemistry Letters, 2008, 37, 1012-1015.

[5] Yahiro, H., Eguchi, Y., Eguchi, K., Arai, H., J. App. Electrochem., 1988, 18, 527-531.

[6] Sammes, N., Du,Y., Fuel Cell Technologies: State and Perspectives., 2005, 19-34.

[7] Steele, B. C. H., Solid State Ionics, 2000, 129, 95-110.

[8] Zhongliang Zhan, T.-L., J. Electrochem. Soc., 2001, 148, A427-A432.

[9] Suzuki, T., Kosacki, I., Anderson, H. U., Solid State Ionics, 2002, 151, 111-121.

[10] Kosacki, I., Suzuki, T., Petrovsky, V. \& Anderson, H. U., Solid State Ionics, 2000,136, 1225-1233.

[11] De Souza, R. A., Pietrowski, M. J., Anselmi-Tamburini, U., Kim, S., Munir, Z. A., Martin, M., Phys. Chem. Chem. Phys., 2008, 10, 2067-2072.

[12] Jin-Phillipp, N. Y., Sata, N., Maier, J., Scheu, C., Hahn, K., Kelsch, M., Ruehle. M., J. Chem. Phys., 2004, 120, 2375-2381.

[13] Korte, C., Schichtel, N., Hesse, D. \& Janek, J. Monatshefte fur Chemie, 2009, 140, 10691080. 
Submitted to

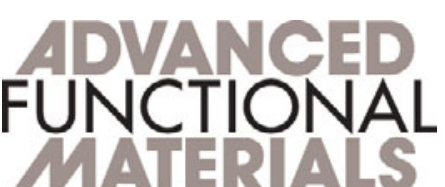

[14] Garcia-Barriocanal, J., Rivera-Calzada, A., Varela, M., Sefrioui, Z. Iborra, E., Leon, C., Pannycook, S. J., Santamaria, J., Science, 2008, 321, 676-680.

[15] Kosacki, I., Accelerated Materials Discovery for Energy Storage and Conversion Devices UK ERC Conference, 2007.

[16] Kosacki, I., Superlattice Electrolyte for Energy Application, Invention Disclosure \#5153.1685, 2006.

[17] Guo, X., Vasco, E., Mi, S., Szot, K., Wachsman, E., Waser, R., Acta Materialia, 2005, $53,5161-5166$.

[18] Fleig, J., Solid State Ionics, 2002, 150, 181-193.

[19] Zha, S., Xia, C., Meng, G., J. Power Sources, 2003, 115, 44-48.

[20] Williams, D. B., Carter, C. B., Transmission Electron Microscopy: A textbook for materials science. Springer, 1996.

[21] Gatan Digital Micrograph. (GMS 1.8), 2009.

[22] Packer, R. J., Ph. D. Thesis, University of London, UK, 2009.

[23] Crank. J., The Mathematics of Diffusion, Oxford University Press, 1975, pp36.

[24] The Mathworks inc. Matlab version 6.5. 2003 Natick.

[25] Saraf, L., Wang, C. M., Shutthanandan, V., Zhang, Y., Marina, O., Baer, D. R., Thevuthasan, S., Nachimuthu, P., Lindle, D. W., J. Mats. Res. 2005, 8, A525-A527.

[26] Barsoum, M. W., Fundamentals of Ceramics, Taylor and Francis Group, Oxon, UK, 2003, pp190.

[27] Migani, A., Neyman, K. M., Illas, F., Bromley, S. T., J. Chem. Phys, 2009, 131,

[28] Wu, L., Wisemann, H. J., Moodenbaugh, A. R., Klie, R. F., Yimie Zhu, Welch, D. O., Suenaga, M., Phys.Rev. B, 2004, 69, 124151-9.

[29] Ye, F., Mori, T., Ou, D. R., Zou, J. \& Drennan, J. Mats. Res. Bulletin, 2007, 42, 943-949.

[30] Scribner Associates inc. ZView version 3.1c. 2007 Southern Pines.

[31] Scott, C. P., Craven, A. J., Hatto, P., Davies, D., J. Microscopy, 2003, 182, 186-191. 